\title{
Ascending thoracic aortic aneurysms are associated with compositional remodeling and vessel stiffening but not weakening in age-matched subjects
}

\author{
Dimitrios C. Iliopoulos, MD, PhD, ${ }^{\mathrm{a}, \mathrm{b}}$ Eleftherios P. Kritharis, BSc, ${ }^{\mathrm{c}}$ Athina T. Giagini, MSc, ${ }^{\mathrm{c}}$ Stavroula A. Papadodima, MD, PhD, ${ }^{\mathrm{d}}$ \\ and Dimitrios P. Sokolis, $\mathrm{PhD}^{\mathrm{c}}$
}

\begin{abstract}
Objective: We sought to examine in age-matched subjects the biomechanical and compositional remodeling associated with ascending thoracic aortic aneurysms according to region and direction.
\end{abstract}

\begin{abstract}
Methods: Whole, fresh, degenerative ascending thoracic aortic aneurysms were taken from 26 patients (age, $69 \pm$ 2 years; maximum aortic diameter, $5.9 \pm 0.3 \mathrm{~cm}$ ) during elective surgical intervention, and 15 nonaneurysmal ascending thoracic aortas were obtained during autopsies (age, $66 \pm 3$ years; maximum aortic diameter, $3.3 \pm$ $0.2 \mathrm{~cm})$. These were cut into anterior, right lateral, posterior, and left lateral regions, and circumferentially and longitudinally oriented specimens were prepared. The aortic specimens were submitted to histomorphometric and biomechanical studies, including measurement of failure strain (ie, extensibility), failure stress (ie, strength), and peak elastic modulus (ie, stiffness).
\end{abstract}

Results: Wall elastin, but not collagen content, decreased in aneurysmal specimens, displaying lower wall thickness and failure strain, higher peak elastic modulus, and equal failure stress than control specimens in the majority of regions and directions. Similar differences were noted in pooled data from all regions. Regional variations in mechanical parameters were mostly found in longitudinally oriented tissue. Circumferential specimens showed higher failure stress and peak elastic modulus but equal failure strain than longitudinal specimens.

Conclusions: Our findings contradict previous studies on ascending thoracic and abdominal aortic aneurysms, suggesting that the former might not cause weakening but rather only stiffening and reduction in tissue extensibility and elastin content. Marked heterogeneity was evident in healthy and aneurysmal aortas. The present data offer insight into the pathogenesis of aneurysm dissection. Information on directional and regional variations is pertinent because dissections develop circumferentially and bulging preferentially occurs in the anterior region.

Ascending thoracic aortic aneurysm (ATAA) is a chronic degenerative pathology that occurs mostly in the elderly, with very high mortality rates. ${ }^{1,2}$ Presently, ATAA formation is thought to be triggered by some localized form of aortic wall injury placed over genetic, age-related, and environmental factors, yet the exact sequence and nature of these events is poorly understood and mostly extrapolated from those of abdominal aortic aneurysms (AAAs). ${ }^{1-3}$ Once aneurysm formation is initiated, it is associated with destructive remodeling of the aortic wall, the time course of which is characteristic of steady structural deterioration, radial enlargement, rearrangement of hemodynamic loads, and ultimately rupture. ${ }^{3-5}$ Aneurysm rupture is a biomechanical failure that occurs when the stresses exerted on the aortic wall by hemodynamic loads exceed the tissue's capacity to

\footnotetext{
From the Laboratory of Experimental Surgery and Surgical Research ${ }^{\mathrm{a}}$ and the Department of Forensic Medicine and Toxicology, ${ }^{\mathrm{d}}$ School of Medicine, University of Athens, Athens, Greece; the Department of Cardiothoracic Surgery, ${ }^{\mathrm{b}}$ Athens Medical Center, Athens, Greece; and the Laboratory of Biomechanics, Foundation of Biomedical Research, Academy of Athens, ${ }^{\text {c }}$ Athens, Greece.

Received for publication March 24, 2008; revisions received May 13, 2008; accepted for publication July 5, 2008.

Address for reprints: Dimitrios P. Sokolis, PhD, 35 Lefkados St, Athens 15354, Greece (E-mail: DimitrisSokolis@ath.forthnet.gr).

J Thorac Cardiovasc Surg 2009;137:101-9

$0022-5223 / \$ 36.00$

Copyright $(C) 2009$ by The American Association for Thoracic Surgery

doi:10.1016/j.jtcvs.2008.07.023
}

sustain stress. ${ }^{6}$ Knowledge of the mechanical properties of aortic aneurysms, together with their individual geometry, is vital in predicting their risk of rupture. ${ }^{6}$

Even though the biomechanical remodeling accompanying AAA formation has been widely investigated (see the review article by Vorp and Vande Geest ${ }^{6}$ and the references listed therein), much less attention has been paid to ATAA. Among the few studies available, Koullias and colleagues ${ }^{7}$ offered an estimate of the in vivo mechanical properties of ATAA, Okamoto and associates ${ }^{8}$ examined the in vitro properties of the dilated ascending aorta, and Vorp and coworkers ${ }^{9}$ compared the biomechanical properties of ATAA with respect to the nonaneurysmal aorta, reporting lower strength and higher stiffness in the former. However, the latter findings might not be appropriate because the 2 subject groups tested were not age matched, and aging reportedly impairs the biomechanical properties and tensile strength of both dilated ${ }^{8}$ and healthy ${ }^{10,11}$ aortas.

Accordingly, this article re-examines the influence of aneurysms on the biomechanical properties of the ascending aorta in age-matched subject groups according to region and direction and reports results contradictory to those of Vorp and coworkers (ie, that ATAAs are stiffer but not weaker compared with healthy aortas). ${ }^{9}$ Our biomechanical studies were coupled with histologic observations quantifying alterations in extracellular matrix composition. Only one 


\section{Abbreviations and Acronyms}

AAA $=$ abdominal aortic aneurysm

ATAA $=$ ascending thoracic aortic aneurysm

study has previously evaluated the effect of ATAA on media composition ${ }^{12}$; we extend those findings by assessing layerspecific alterations in different aortic regions.

\section{MATERIALS AND METHODS \\ Human Aortic Tissue and Specimen Preparation}

Whole degenerative ATAAs were harvested fresh from patients $(n=26)$ during elective surgical repair at the Department of Cardiothoracic Surgery of the Athens Medical Center. Whole nonaneurysmal ascending thoracic aortas (control specimens) were harvested within 24 hours of death from subjects $(n=15)$ undergoing autopsy at the Department of Forensic Medicine and Toxicology of the Athens University Medical School. ATAAs associated with aortic dissection and Marfan syndrome were excluded from the protocol, as were aortas from young adults $(<40$ years old). Clinical data were taken from the patients' hospital charts and cadaveric data during autopsy. The ATAA's maximum preoperative diameter was recorded, as reported by the surgeons or as evaluated during the echocardiographic examination, magnetic resonance imaging, or computed tomographic scanning. All tissues were excised as short tubes from above the sinuses of Valsalva, immersed in saline solution, and kept in a refrigerator at $4^{\circ} \mathrm{C}$.

Tissues were submitted to histologic processing and biomechanical tests within 24 hours after harvesting. First, the tubes were cleaned of all adherent tissues and cut into 4 regions (ie, anterior, posterior, and left and right lateral) with respect to circumferential position. Multiple specimens with circumferential and longitudinal direction were taken from each region with a standardized dog-bone shape for biomechanical testing, as in our recent study, ${ }^{13}$ whereas a circumferential specimen from each region was kept for histomorphometry. The research protocol was approved by the Institutional Ethics Committee on Human Research. Informed consent was obtained from the patients and from relatives for the cadaveric study subjects.

\section{Histomorphometric Studies}

Aortic specimens were fixed with $10 \%$ buffered formalin over 24 hours, dehydrated in graded ethanol and xylol, and embedded in paraffin wax. Serial 5- $\mu \mathrm{m}$-thick sections were cut and treated with hematoxylin and eosin for examination of overall vessel morphology, orcein for elastin, and Sirius red for collagen staining. Histologic slides were assessed blindly, as previously described by our group. ${ }^{14}$ Briefly, polychromatic images were taken with a digital camera (Altra20; Soft Imaging System, Munster, Germany) fitted to an optical microscope (Olympus CX31; Olympus, Tokyo, Japan) and processed with commercial image-analysis software (Image-Pro Plus v4.5; Media Cybernetics, Inc, Silver Spring, Md). Thickness measurements of the tunica intima, media, and adventitia and of the entire wall were performed in 10 representative positions, and an average was calculated. Elastin and collagen area densities of the different layers and the entire vessel wall were also measured in 10 representative positions with respect to the total wall area in the region of interest after the micrographs were segmented with the software. Values for each specimen were averages from 3 sections.

\section{Biomechanical Studies}

The ascending aortic specimens were individually submitted to biomechanical tests on a tensile tester (Vitrodyne V1000 Universal Tester; Liveco, Inc, Burlington, Vt), as previously described by our group. ${ }^{13,14}$ They were clamped by using the apparatus grips, with sandpaper used to avoid slip-

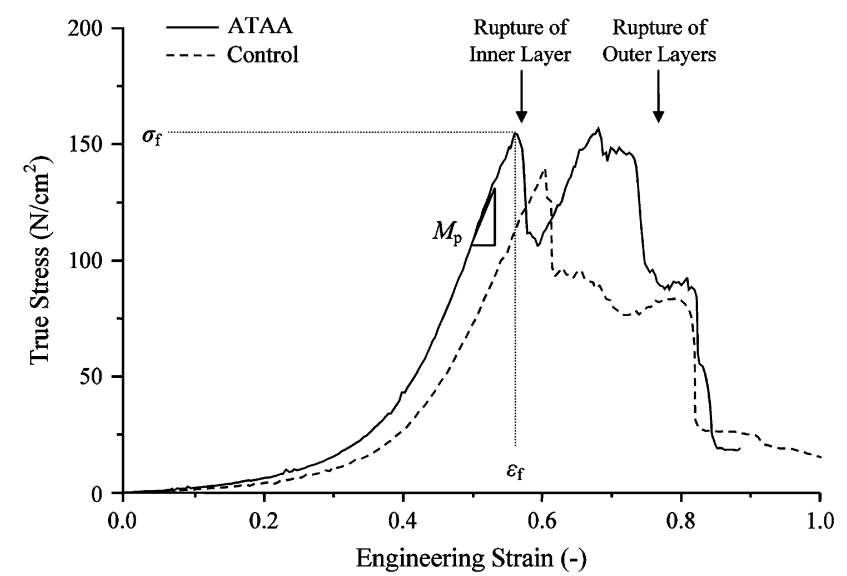

FIGURE 1. Typical stress-strain curves of a control and ascending thoracic aortic aneurysm $(A T A A)$ specimen showing multiple aortic ruptures. The symbols $\sigma_{\mathrm{f}}, \epsilon_{\mathrm{f}}$, and $\mathrm{M}_{\mathrm{p}}$ denote failure stress, failure strain, and peak elastic modulus for the ATAA specimen.

page, and immersed in physiologic saline regulated to $37^{\circ} \mathrm{C}$ with a heater coil (1130A; PolyScience, Niles, Ill). Their width and thickness were measured noncontacting by using a laser micrometer (LS-3100; Keyence Corp, Osaka, Japan) with 1- $\mu \mathrm{m}$ resolution; 4 measurements were taken because of the nonuniform specimen geometry, and the mean values were recorded.

The lower grip of the apparatus was stationary, whereas the upper grip was set to move at a $20 \%$ per minute strain rate until rupture of the specimens. An experiment was considered successful only if the specimen ruptured in the narrowed region. Most specimens displayed multiple ruptures, usually 2 and sometimes 3 , because the inner layers of the aortic wall ruptured before the outer layers (Figure 1). Data were recorded every 0.1 seconds (50-Hz sampling frequency). The load cell (GSO-500; Transducer Techniques, Temecula, Calif) fitted to the tensile tester came with a $500-\mathrm{g}$ capacity and a 0.25 -g resolution, and a pair of sonometrics piezoelectric crystals (Sonometrics Corp, London, Ontario, Canada) sutured on the tissue measured extension in the narrowed region by using principles of ultrasonography with $1-\mu \mathrm{m}$ accuracy.

\section{Data Processing}

True stress was calculated as applied load divided by the current (deformed) cross-sectional area; the latter was calculated based on the tissue incompressibility assumption as initial (undeformed) specimen volume divided by the current specimen length. Failure stress, an index of tissue strength, was taken as maximum stress before the specimen's first rupture (Figure 1). Engineering strain was calculated by subtracting unity from the deformed/initial length ratio. Failure strain, an index of tissue extensibility, was taken as the strain at rupture. Elastic modulus was calculated as the slope of the stress-strain curve, and peak elastic modulus, an index of maximum tissue stiffness, was taken as the highest value before the first rupture.

The 2-tailed unpaired Student's $t$ test was used to determine aneurysmbased (ATAA vs control groups) and directional-related differences. Oneway analysis of variance, followed by the Tukey post-hoc test, was used for regional differences. Spearman rank order correlation coefficients were used to describe correlations. Commercially available software (SPSS v12.0; SPSS, Inc, Chicago, Ill) was used for all analyses.

\section{RESULTS}

No differences were noted in age ( $69 \pm 2$ vs $66 \pm 3$ years $[$ mean $\pm \mathrm{SEM}], P>.2)$ and male/female ratio (17/9 vs $10 / 5)$ between the 2 subject groups, whereas aortic diameter was 
TABLE 1. Categorization of aortic specimens submitted to biomechanical testing into groups according to pathology, region, and direction

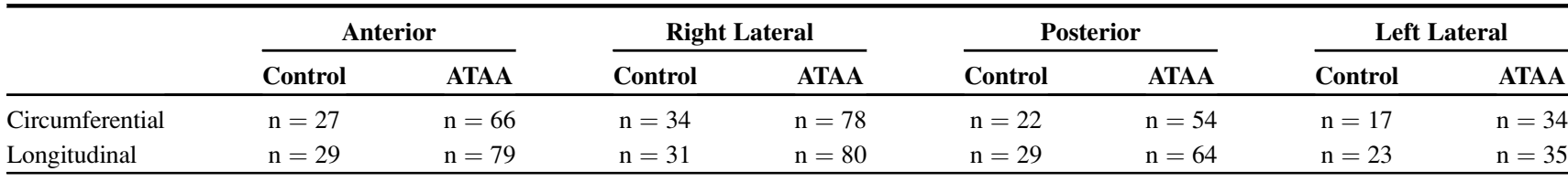

ATAA, Ascending thoracic aortic aneurysm; $n$, number of specimens that fractured in the narrowed region.

significantly higher in patients with ATAAs compared with autopsy subjects $(5.9 \pm 0.3$ vs $3.3 \pm 0.2 \mathrm{~cm}, P<.001)$. The aortic specimens submitted to biomechanical testing were categorized into groups according to pathology, region, and direction (Table 1). Less than $3 \%$ of the total number was excluded from analysis because of slippage at the grips or nonfailure in the narrowed region.

Histomorphometric parameters for ATAA and control specimens from the different aortic regions and for pooled data from all regions considered together are listed in Table 2, with Figure 2 showing representative histologic sections. Compared with control specimens, elastin area density decreased in tunica intima, media, and the entire wall of the ATAA specimens in every region and for pooled data. The same was true for collagen density in the media of ATAA specimens while increasing in the adventitia and remaining invariant in the entire wall for pooled data. Intimal thickness of the ATAA specimens was higher than that of control specimens in some regions and pooled data, media thickness was lower, and adventitia and wall thickness were unchanged. No regional variations were found for ATAA or control specimens.

Stress-strain curves of all specimens from the anterior region tested successfully are shown in Figure 3, exhibiting marked variations. The central body of control curves was displaced rightward from that of ATAA curves, and curves for the circumferential direction were on average displaced upward from those for the longitudinal direction. Similar differences among curves were observed in the remaining regions.

In contrast to our histologic data, measurements on fresh tissue indicated that ATAA specimens displayed lower wall thickness than control specimens, with region having no influence (Figure 4). The failure strain of ATAA specimens was lower than that of control specimens in several regions and directions; no directional variations were found for either ATAA specimens or control specimens (Figure 5). Regional variations for control specimens were nonsignificant, whereas for ATAA specimens, failure strain of the left lateral region was higher than that of the anterior, right lateral, and posterior regions in both circumferential and longitudinal specimens.

Failure stress of ATAA specimens did not differ from that of control specimens in all but the left lateral region in the longitudinal direction (Figure 6). Regional variations were nonsignificant for both tissue types in the circumferential direction; in the longitudinal direction failure stress of the right lateral region was higher than that of the anterior and

TABLE 2. The effect of aneurysm (ATAA vs control specimen) and region (anterior vs right lateral vs posterior vs left lateral) on histomorphometric parameters of the aortic wall and its layers

\begin{tabular}{|c|c|c|c|c|c|c|c|c|c|c|c|}
\hline & & \multicolumn{2}{|c|}{ Anterior } & \multicolumn{2}{|c|}{ Right Lateral } & \multicolumn{2}{|c|}{ Posterior } & \multicolumn{2}{|c|}{ Left Lateral } & \multicolumn{2}{|c|}{ Pooled Data } \\
\hline & & $\begin{array}{l}\text { Control } \\
(n=15)\end{array}$ & $\begin{array}{c}\text { ATAA } \\
(n=26)\end{array}$ & $\begin{array}{l}\text { Control } \\
(n=15)\end{array}$ & $\begin{array}{c}\text { ATAA } \\
(n=26)\end{array}$ & $\begin{array}{l}\text { Control } \\
(n=15)\end{array}$ & $\begin{array}{c}\text { ATAA } \\
(n=26)\end{array}$ & $\begin{array}{l}\text { Control } \\
(n=15)\end{array}$ & $\begin{array}{c}\text { ATAA } \\
(n=26)\end{array}$ & $\begin{array}{r}\text { Control } \\
(n=60)\end{array}$ & $\begin{array}{c}\text { ATAA } \\
(n=104)\end{array}$ \\
\hline \multirow{4}{*}{$\begin{array}{r}\text { Elastin } \\
(\%)\end{array}$} & I & $30.1 \pm 4.8$ & $.9 \pm 2.3$ & $35.1 \pm 5.4$ & $19.0 \pm$ & $.7 \pm 6.6$ & $15.8 \pm$ & $31.2 \pm 5.2$ & .7 & 2.7 & \pm \\
\hline & M & $3 \pm 2.0$ & 1 & & 1 & 1.8 & $16.9 \pm$ & $26.2 \pm 1.8$ & $19.4 \pm$ & 1.0 & \pm 0 \\
\hline & A & $4.8 \pm 0.8$ & $11.0 \pm 2.2$ & $8.1 \pm 2.1$ & $10.1 \pm 2.0$ & $8.8 \pm 2.0$ & $10.2 \pm 1.4$ & $12.6 \pm 2.1$ & $7.9 \pm 0.9$ & $8.6 \pm 0.7$ & $9.9 \pm 0$ \\
\hline & W & $21.5 \pm 1.9$ & $14.1 \pm 1.4$ & $23.2 \pm 2.1$ & $15.0 \pm 1.0$ & $5 \pm 1.9$ & $14.4 \pm 1.3$ & $25.3 \pm 1.9$ & $15.5 \pm$ & 1.0 & \pm 0 \\
\hline \multirow{4}{*}{$\begin{array}{c}\text { Collagen } \\
(\%)\end{array}$} & I & $22.7 \pm 4.1$ & $22.3 \pm 3.5$ & $20.5 \pm 4.7$ & $20.4 \pm 2.6$ & $17.3 \pm 3.7$ & $20.2 \pm$ & $15.7 \pm$ & $24.0 \pm 3.0$ & $19.0 \pm 1.8$ & \pm 1 \\
\hline & M & $31.6 \pm 2.3$ & $20.3 \pm 1.7$ & $30.3 \pm 2.3$ & $22.0 \pm 1.7$ & $29.8 \pm 2.2$ & $22.6 \pm 1.6$ & $29.0 \pm 2.1$ & $22.7 \pm 1.7$ & $30.1 \pm 1.1$ & $21.9 \pm 0$ \\
\hline & A & $39.8 \pm 3.8$ & $43.0 \pm 2.2$ & $35.7 \pm 3.0$ & $40.6 \pm 2.7$ & $32.8 \pm 4.4$ & $40.8 \pm$ & $33.0 \pm 3.2$ & $39.3 \pm 3.1$ & $35.3 \pm 1.8$ & $40.9 \pm$ \\
\hline & $\mathrm{W}$ & $32.4 \pm 2.0$ & $28.8 \pm 1.1$ & $31.0 \pm 1.9$ & $27.1 \pm$ & $9 \pm 2.3$ & $28.5 \pm$ & $29.4 \pm 1.9$ & $28.7 \pm 1.6$ & 30.5 & $28.5 \pm 0$ \\
\hline \multirow{4}{*}{$\begin{array}{l}\text { Thickness } \\
(\mu \mathrm{m})\end{array}$} & I & $101 \pm 13$ & $250 \pm 53$ & $99 \pm 18$ & $186 \pm 39$ & $100 \pm 18$ & $256 \pm 50$ & $87 \pm 13$ & $240 \pm 49$ & $97 \pm 8$ & $233 \pm$ \\
\hline & M & $1320 \pm 62$ & $1079 \pm 78$ & $1241 \pm 72$ & $1140 \pm 123$ & $1237 \pm 50$ & $1076 \pm 65$ & $1486 \pm 93$ & $1134 \pm 96$ & $1322 \pm 38$ & $1107 \pm$ \\
\hline & A & $299 \pm 46$ & $292 \pm 48$ & $346 \pm 31$ & $262 \pm 33$ & $332 \pm 44$ & $322 \pm 45$ & $273 \pm 64$ & $263 \pm 42$ & $312 \pm 23$ & $285 \pm 2$ \\
\hline & $\mathrm{W}$ & $1720 \pm 53$ & $1621 \pm 93$ & $1687 \pm 86$ & $1588 \pm 119$ & $1669 \pm 64$ & $1654 \pm 79$ & $1846 \pm 100$ & $1637 \pm 80$ & $1731 \pm 39$ & $1625 \pm 4$ \\
\hline
\end{tabular}

Values are presented as means \pm the standard error of the mean. ATAA, Ascending thoracic aortic aneurysm; $n$, number of specimens; $I$, intima; $M$, media; $A$, adventitia; $W$, wall. Control versus ATAA specimens: elastin—anterior region, $P=.001(\mathrm{I}), P=.01(\mathrm{M}), P=.05(\mathrm{~A}), P=.003(\mathrm{~W})$; right lateral region, $P=.002(\mathrm{I}), P=.002(\mathrm{M}), P<.001(\mathrm{~W})$; posterior region, $P<.001(\mathrm{I}), P=.004(\mathrm{M}), P=.003(\mathrm{~W})$; left lateral region, $P=.03(\mathrm{I}), P=.01(\mathrm{M}), P=.05(\mathrm{~A}), P<.001(\mathrm{~W})$; pooled data, $P<.001(\mathrm{I}), P=.01(\mathrm{M}), P<.001$ (W). Collagen-anterior region, $P<.001(\mathrm{M})$; right lateral region, $P=.006(\mathrm{M})$; posterior region, $P=.01(\mathrm{M})$; left lateral region, $P=.03(\mathrm{M})$; pooled data, $P<.001(\mathrm{M}), P=.02$ (A). Thickness-anterior region, $P=.05(\mathrm{I}), P=.05(\mathrm{M})$; posterior region, $P=.04(\mathrm{I}), P=.05(\mathrm{M})$; left lateral region, $P=.03$ (I), $P=.02$ (M); pooled data, $P<.001$ (I), $P=.002$ (M). Other differences were nonsignificant. Regional differences were nonsignificant for control specimens and ATAA specimens. 

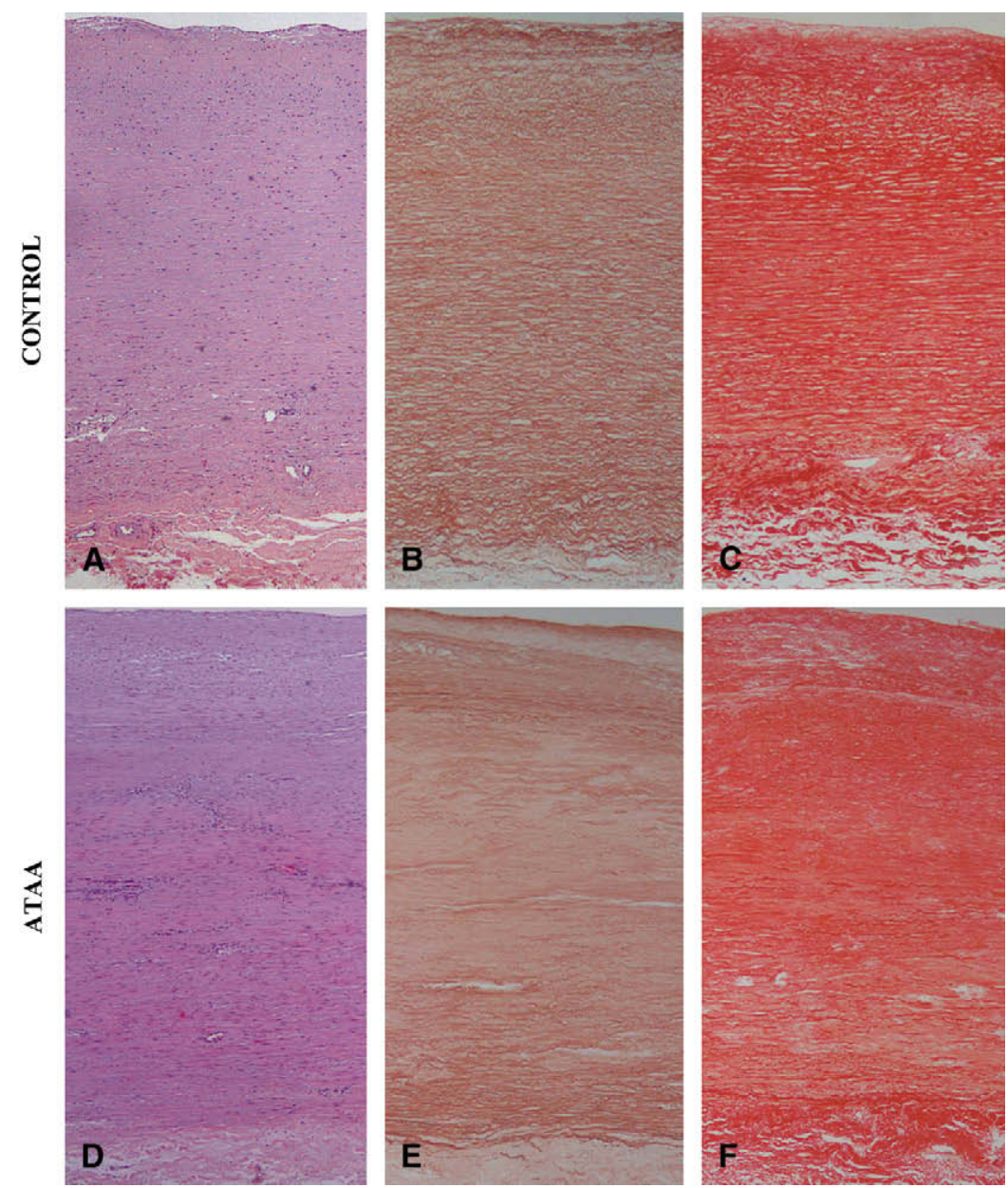

FIGURE 2. Representative histologic sections of a control (upper panel) and ascending thoracic aortic aneurysm (ATAA) specimen (lower panel) stained with hematoxylin and eosin for examination of overall vessel wall morphology (A and D), orcein for elastin staining (B and E), and Sirius red for collagen staining (C and F). (Original magnification $4 \times$ ).

posterior regions for ATAA specimens and than that of the anterior and left lateral regions for control specimens. Furthermore, the failure stress of circumferential specimens from a given region was significantly higher than that of the corresponding longitudinal specimens.

The peak elastic modulus of ATAA specimens was higher than that of control specimens in most regions and directions (Figure 7). Regional and directional differences were similar for both ATAA specimens and control specimens. No regional differences were detected in the circumferential direction, whereas the right lateral region showed the highest peak elastic modulus among all regions in the longitudinal direction. As with failure stress, peak elastic modulus was higher in the circumferential direction.

Failure stress (circumferential, $R=-0.22$ and $P<.001$; longitudinal, $R=-0.14$ and $P=.02$ ) and peak elastic modulus (circumferential, $R=-0.21$ and $P=.001$; longitudinal, $R=-0.16$ and $P=.01$ ) correlated negatively but weakly with ATAA diameter for pooled data. Negative and stronger were the correlations of those parameters with wall thickness measured on fresh tissue (circumferential, $R=-0.42$ and $P<.001$; longitudinal, $R=-0.33$ and $P<.001$; and circumferential, $R=-0.48$ and $P<.001$; longitudinal, $R=-0.38$ and $P<.001$, respectively). Coefficients were lower when each region was examined individually (data not shown). Correlations of failure strain with ATAA diameter and wall thickness did not reach significance in either direction. Also nonsignificant were the correlations among all 3 biomechanical parameters and aortic diameter or wall thickness for control tissue, as regards pooled data from all regions and from each region separately (data not shown). Thickness correlated weakly with ATAA specimen $(R=0.16, P<.001)$ and control specimen $(R=-0.14, P=.04)$ diameter for pooled data, albeit in an opposite fashion. Correlations were nonsignificant within most aortic regions (data not shown).

Analogous variations in failure properties among ATAA specimens and control specimens were noted when data from all regions were pooled (Figure 8). These results suggest that the ATAA tissue was stiffer and less extensible 

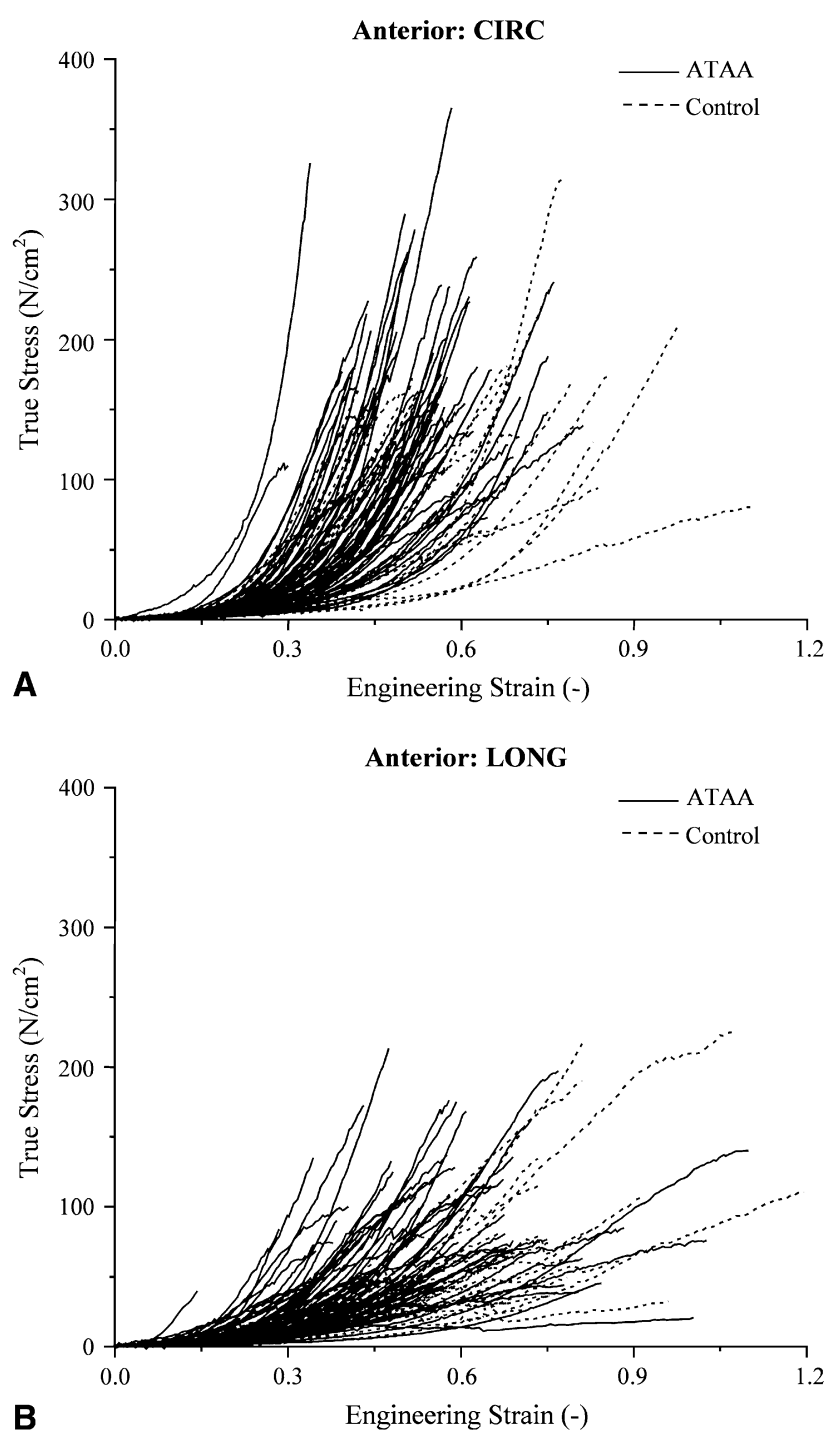

FIGURE 3. Stress-strain curves of all specimens from the anterior region with circumferential $(C I R C ; \mathrm{A})$ and longitudinal $(L O N G ; \mathrm{B})$ orientation. $A T A A$, Ascending thoracic aortic aneurysm.

but equally strong in circumferential specimens if not stronger as in longitudinal specimens compared with control tissue. Comparisons at the same thickness level through the linear regressions of biomechanical properties with aortic wall thickness confirmed the abovementioned results. Identical directional differences were observed for both tissues, with circumferential specimens being stronger and stiffer but equally extensible than longitudinal specimens.

\section{DISCUSSION}

It was unexpected to find that the failure stress (ie, tensile strength) of ATAA specimens did not vary or was even higher than that of nonaneurysmal tissue because it is generally acknowledged that aortic aneurysms rupture as the tissue becomes progressively weaker. ${ }^{6}$ Our finding is in

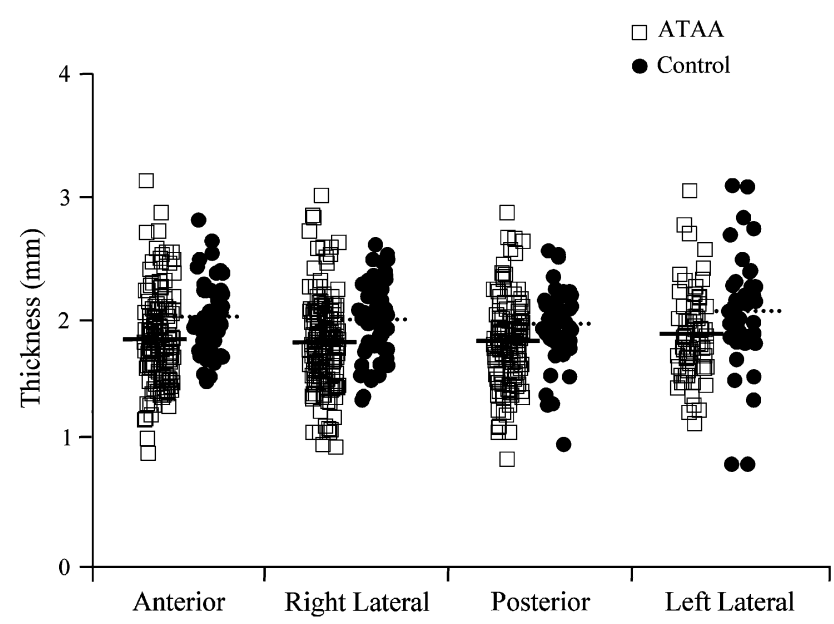

FIGURE 4. Wall thickness of ascending thoracic aortic aneurysm ( $A T A A$; open squares) and control (filled circles) tissue specimens from the anterior, right lateral, posterior, and left lateral regions with both circumferential and longitudinal directions. Data are individual measurements with means, with horizontal continuous lines denoting ATAA specimens and dotted lines denoting control specimens. Control versus ATAA specimens: anterior region, $P=.002$; right lateral region, $P<.001$; posterior region, $P=.02$; left lateral region, $P=.02$. Regional differences were nonsignificant for ATAA and control specimens.

marked contrast with the results reported by Vorp and coworkers, ${ }^{9}$ who compared control and ATAA tissue and found that the former was stronger than the latter in both circumferential and longitudinal directions. Compared with that study, we have tested considerably more specimens for both subject groups, and comparisons were performed among specimens taken from the same region. Most importantly, the 2 subject groups were age matched in our study, whereas in the study by Vorp and coworkers ${ }^{9}$ the mean age of the control group was 15 years less than that of the ATAA group. This might explain the inconsistency among the 2 studies because there has been strong evidence from many reports $8,10,11,15-17$ that the mechanical properties and strength of aortic wall tissue show pronounced age dependency. In particular, Okamoto and colleagues ${ }^{8}$ reported a negative correlation of failure stress with age for the dilated ascending thoracic aorta, whereas Groenink and associates ${ }^{10}$ and Mohan and Melvin ${ }^{11,15}$ documented the same finding for the healthy descending thoracic aorta. Other studies also disclosed that the aorta becomes less extensible ${ }^{16}$ and stiffer ${ }^{17}$ as a result of aging, underscoring the necessity of examining ATAA tissue by using nonaneurysmal agematched tissue as a reference.

Raghavan and associates ${ }^{18}$ found comparable aneurysminduced reductions in failure stress of AAA wall tissue, but again the subject groups examined were not age matched (control group $=47$ years vs AAA group $=69$ years). Furthermore, their findings might not be directly extrapolated to ATAAs because of wide differences in the 2 vessels in terms 


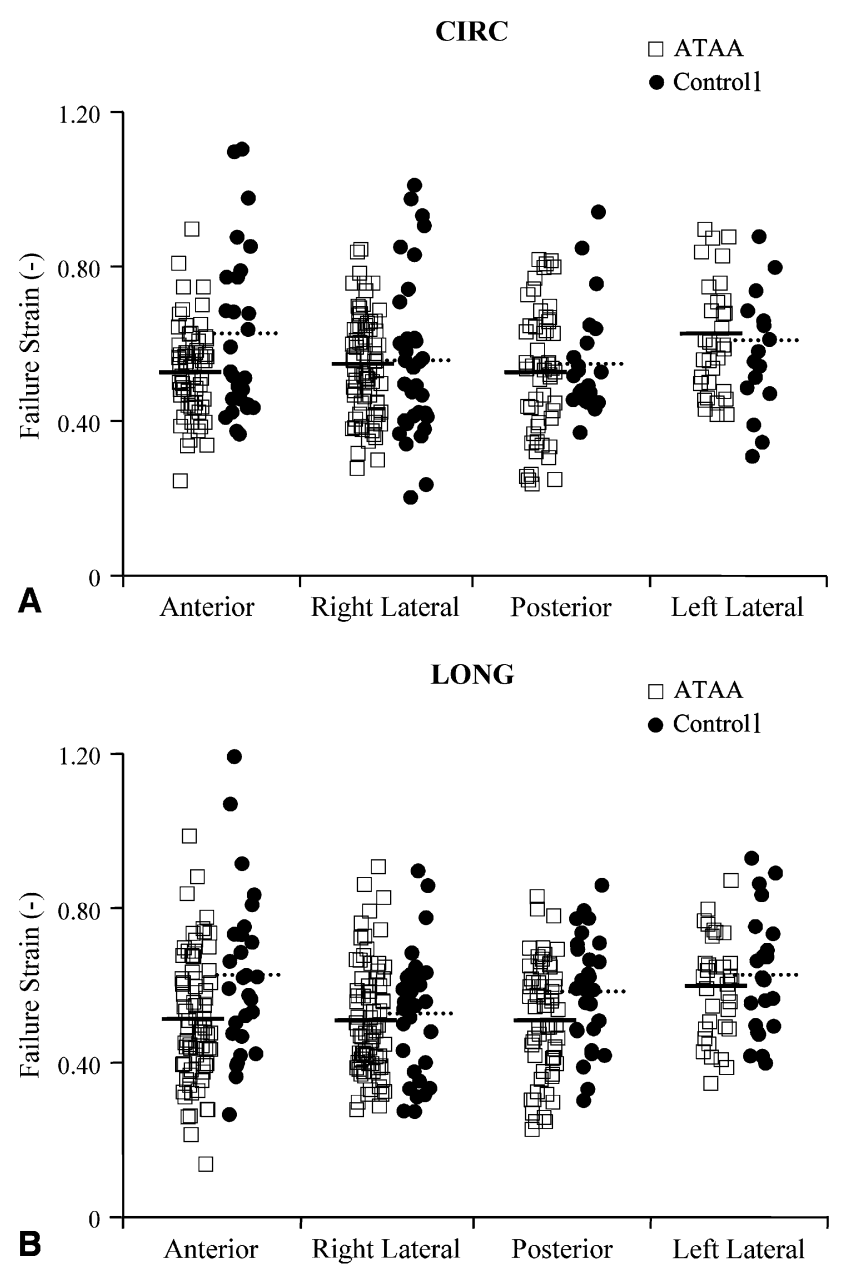

FIGURE 5. Failure strains of ascending thoracic aortic aneurysm (ATAA; open squares) and control (filled circles) tissue specimens from the anterior, right lateral, posterior, and left lateral regions for the circumferential (CIRC; A) and longitudinal $(L O N G$; B) directions. Data are individual measurements with means, with horizontal continuous lines denoting ATAA specimens and dotted lines denoting control specimens. Control versus ATAA specimens: CIRC—anterior region, $P=.009$; LONG—anterior region, $P=.003$; posterior region, $P=.04$. Regional differences: ATAA CIRCanterior versus left lateral region, $P=.001$; right versus left lateral region, $P=.007$; posterior versus left lateral region, $P=.007$. ATAA LONGanterior versus left lateral region, $P=.01$; right versus left lateral region, $P=.002$; posterior versus left lateral region, $P=.007$. Control CIRC and LONG: nonsignificant differences. LONG versus CIRC: nonsignificant differences for ATAA and control specimens. Other differences were nonsignificant.

of hemodynamic conditions, periaortic tissues, embryologic origin of smooth muscle cells, and the different risk between AAAs and ATAAs. ${ }^{2}$

We found that ATAA specimens were significantly stiffer than control specimens in the longitudinal direction, whereas there was a trend for circumferential specimens, but differences were not significant in all regions. Still, the peak elastic modulus of pooled data from all regions was higher in ATAA specimens than in control specimens in both directions.
CIRC

$\square$ ATAA

- Control

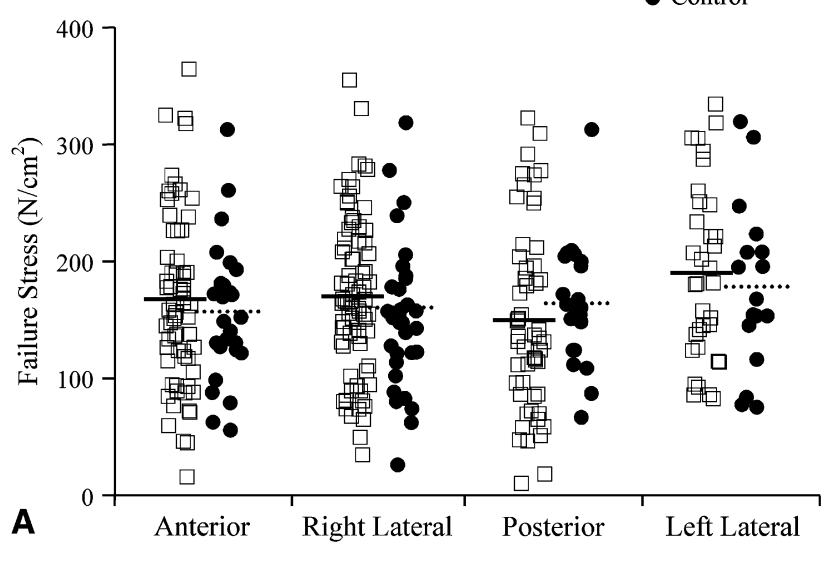

LONG

$\square$ ATAA

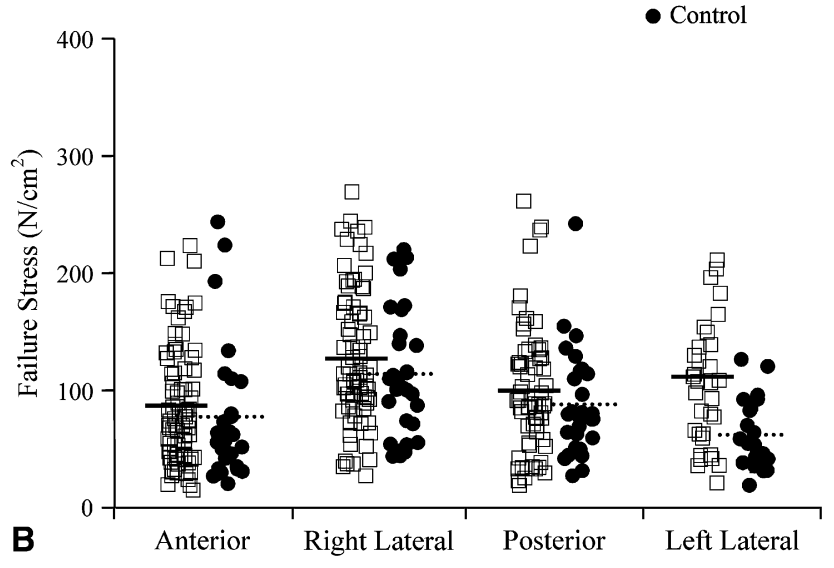

FIGURE 6. Failure stresses of ascending thoracic aortic aneurysm (ATAA; open squares) and control (filled circles) tissue specimens from the anterior, right lateral, posterior, and left lateral regions for the circumferential (CIRC; A) and longitudinal $(L O N G$; B) directions. Data are individual measurements with means, with horizontal continuous lines denoting ATAA specimens and dotted lines denoting control specimens. Control versus ATAA specimens: CIRC, nonsignificant differences; LONG-left lateral region, $P=.003$. Regional differences: ATAA CIRC, nonsignificant differences; ATAA LONG-anterior versus right lateral region, $P<.001$; right lateral versus posterior region, $P=.003$; control CIRC: nonsignificant differences; control LONG-anterior versus right lateral region, $P=.01$; right versus left lateral region, $P<.001$. LONG versus CIRC: ATAA—anterior region, $P<.001$; right lateral region, $P<.001$; posterior region, $P<.001$; left lateral region, $P<.001$. Control-anterior region, $P<.001$; right lateral region, $P=.008$; posterior region, $P<.001$; left lateral region, $P<.001$. Other differences were nonsignificant.

Findings similar to those of the present study have been previously reported for ATAAs in the in vitro study of Vorp and coworkers ${ }^{9}$ and the in vivo study of Koullias and colleagues ${ }^{7}$ and for AAAs in the majority of studies. ${ }^{6,19,20}$

Limited data concerning failure strain of the ascending aorta have been published. The report by Okamoto and associates ${ }^{8}$ for the dilated ascending aorta for patients who were age matched to our ATAA patient group is in agreement 

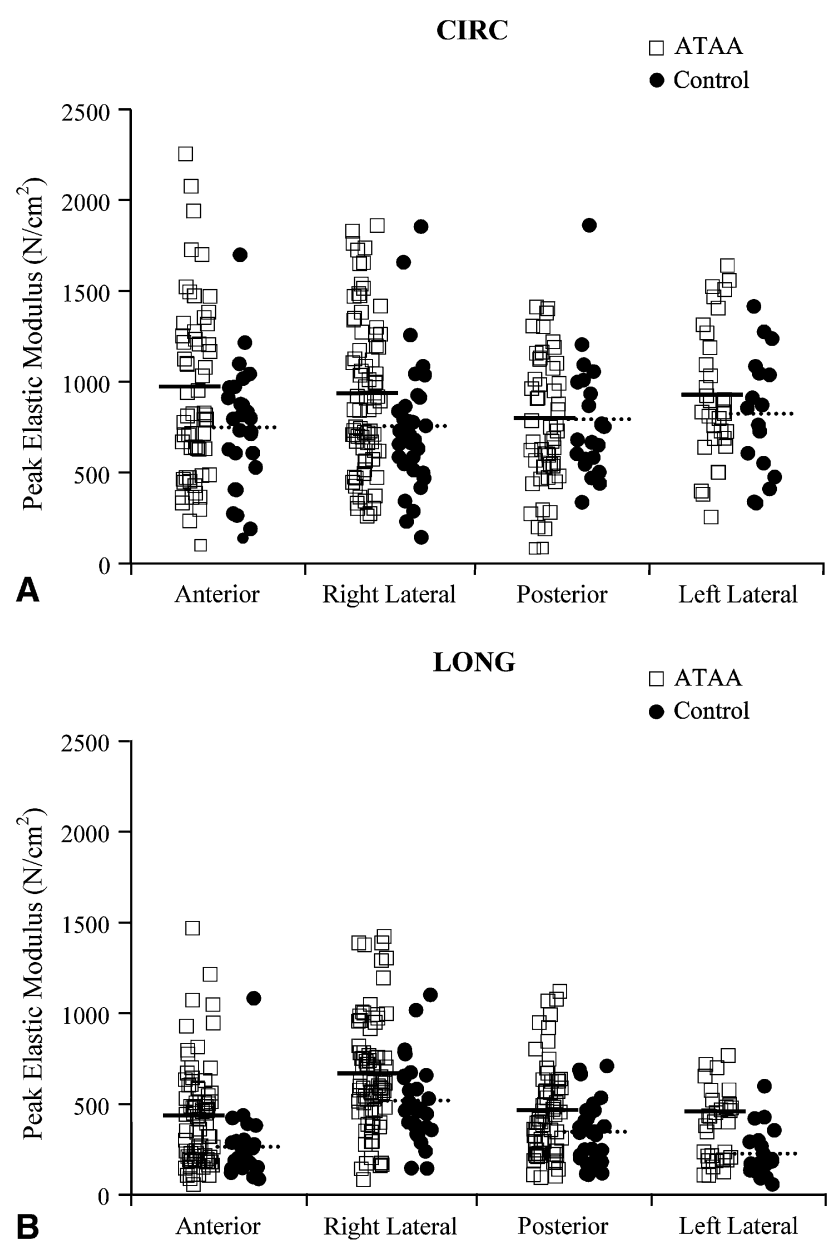

FIGURE 7. Peak elastic modulus of ascending thoracic aortic aneurysm (ATAA; open squares) and control (filled circles) tissue specimens from the anterior, right lateral, posterior, and left lateral regions for the circumferential $(C I R C ; \mathrm{A})$ and longitudinal $(L O N G$; B) directions. Data are individual measurements with means, with horizontal continuous lines denoting ATAA specimens and dotted lines denoting control specimens. Control versus ATAA specimens: CIRC—right lateral region, $P=.03$; LONG—anterior region, $P=.003$; right lateral region, $P=.02$; posterior region, $P=.02$; left lateral region, $P=.004$. Regional differences: ATAA CIRC, nonsignificant differences; ATAA LONG - anterior versus right lateral region, $P<.001$; right lateral versus posterior region, $P<.001$; right versus left lateral region, $P=.002$; control CIRC: nonsignificant differences; control LONG-anterior versus right lateral region, $P<.001$; right lateral versus posterior region, $P=.001$; right versus left lateral region, $P<.001$. LONG versus CIRC: ATAA — anterior region, $P<.001$; right lateral region, $P<.001$; posterior region, $P<.001$; left lateral region, $P<.001$; controlanterior region, $P<.001$; right lateral region, $P=.002$; posterior region, $P<.001$; left lateral region, $P<.001$. Other differences were nonsignificant.

with our data. We found that the nonaneurysmal tissue was more extensible than ATAA tissue in the longitudinal direction, whereas less marked differences were noted in the circumferential direction. Analogous results have been reported earlier by the greater part of biomechanical studies in AAAs, ${ }^{19,20}$ without, however, making distinctions about directions. Taken together, the results on failure stresses and strains help explain why peak elastic modulus was higher in ATAA specimens, whereas failure stresses were equal in the 2 types of tissue. ATAA specimens reached equally maximum stresses at lower strains, so that the peak slopes of their stress-strain curves had to be higher compared with those of healthy specimens.

Our results, displaying directional differences in tensile strength and maximum tissue stiffness, do not corroborate those of Vorp and coworkers, ${ }^{9}$ who found nonsignificant variations in either ATAA or control tissue. However, almost all biomechanical studies on healthy and aneurysmal aortas, including our recent study, ${ }^{13}$ have documented different properties in the circumferential and longitudinal directions. In particular, testing of human healthy descending thoracic aortas under different loading scenarios (ie, uniaxial, ${ }^{11}$ biaxial, ${ }^{15}$ and inflation ${ }^{10}$ ) and inflation tests of porcine healthy descending thoracic aortas ${ }^{21}$ have indicated a preferential circumferential rupture, with the longitudinal direction showing lower failure stress, which is consistent with our data.

This study reports also on the regional heterogeneity of ATAAs and nonaneurysmal aortas. Circumferential specimens showed uniform properties across the vessel circumference, whereas longitudinal specimens from the anterior region were the weakest and least-stiff specimens of all regions examined, as we recently showed. ${ }^{13}$ Regarding the nonaneurysmal aorta, few data are available for the porcine healthy ascending aorta, ${ }^{22}$ according to which, and as with this study, the anterior was the least-stiff aortic region. The heterogeneity in biomechanical properties of AAAs has been reported before by several groups ${ }^{19,23}$ (also see our recent article $^{13}$ ). We examined an anterior, a posterior, and 2 lateral regions of ATAAs, as Thubrikar and associates ${ }^{23}$ did for AAAs. These authors presented similar findings for longitudinal specimens from the anterior region and comparable directional variations for strength and stiffness but did not perform statistical evaluation because of the limited number of specimens tested.

Our histomorphometric studies suggest that the intima was thicker, the media thinner, and the adventitia similar in ATAA tissue than in control tissue. The values of wall thickness measured histologically for ATAA tissue were smaller than those for healthy tissue but not significantly so, which is at some variance with what we measured on fresh tissue, for which wall thickness was significantly reduced with ATAA formation. Discrepancies in the results obtained by means of the 2 methods of thickness measurement can be attributed to the effects of fixation and histologic preparation on aortic dimensions; it is also possible that the significance of thickness differences in fresh tissue was attained with the much larger number of specimens examined. Overall, as evidenced by our study, the tissue undergoes major wall remodeling on diameter enlargement, with the decrease in media partly compensated by an 

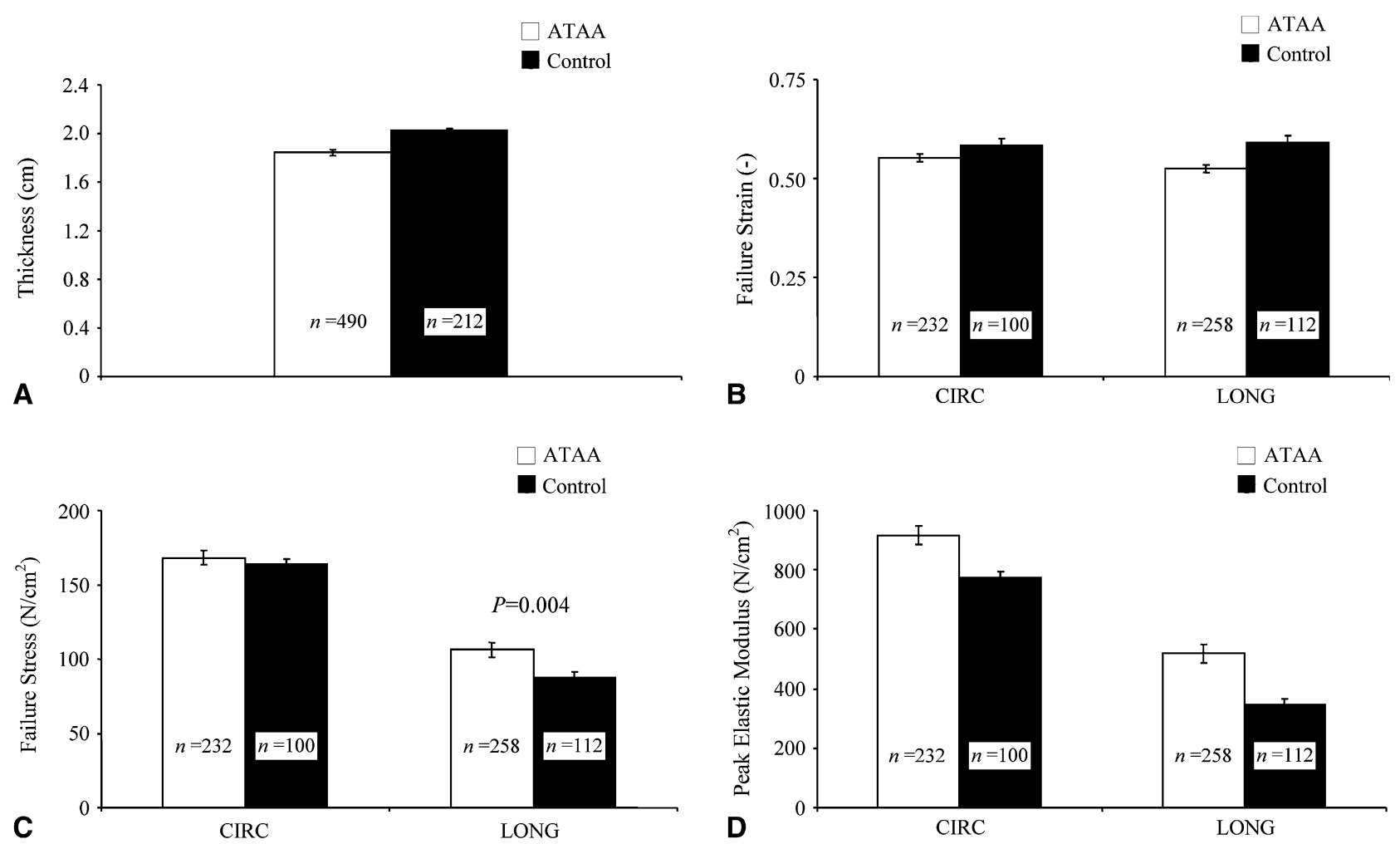

FIGURE 8. Thickness (A), failure strain (B), failure stress (C), and peak elastic modulus (D) of ascending thoracic aortic aneurysm (ATAA; open columns) and control (filled columns) tissue specimens with circumferential (CIRC) and longitudinal (LONG) directions for pooled data from all regions. Values are presented as means \pm standard error of the mean. Control versus ATAA specimens: thickness, $P<.001$; failure strain CIRC, $P=.05$; failure strain LONG, $P<.001$; failure stress LONG, $P=.004$; peak elastic modulus CIRC, $P=.008$; peak elastic modulus LONG, $P<.001$. LONG versus CIRC: failure stress ATAA, $P<.001$; failure stress control, $P<.001$; peak elastic modulus ATAA, $P<.001$; peak elastic modulus control, $P<.001$. Other differences were nonsignificant.

increase in intima thickness. Aortic wall thickness measured on fresh tissue showed a weak inverse correlation with diameter in nonaneurysmal aortas and a weak direct correlation in ATAAs, the latter association underscoring that diameter enlargement of ATAAs might not be associated with vessel wall thinning. The present findings coincide with those of Tang and coworkers. ${ }^{12}$

The study of Tang and coworkers ${ }^{12}$ also investigated the microstructure of collagen and elastin fibers in ATAAs, reporting dissimilar alterations in extracellular matrix composition of the media layer compared with that seen in AAAs. ${ }^{24}$ Similarly to that study, ${ }^{12}$ we found that elastin and collagen area densities decreased in the media layer of ATAA specimens compared with control specimens. We also report a decreased elastin density in the intima and entire wall of ATAA specimens for all regions and an increased collagen density in the adventitia and an invariant density in the entire wall.

Aneurysm formation has been intimately connected with alterations in the histologic structure of aortic tissue, with loss of elastin implicated for dilation and collagen degradation implicated for rupture. ${ }^{25}$ Elastin and collagen are major determinants of the passive mechanical properties of the aortic wall, with extensibility depending on elastin content and microstructure and peak aortic stiffness and tensile strength depending on collagen. ${ }^{26}$ Our histologic findings of considerably decreased wall elastin content substantiate the deficient extensibility of ATAAs, whereas the nonexistent variations in wall collagen content substantiate the similar strength of ATAAs and normal aortas. The increased stiffness at high strains might be associated with elastin fragmentation and earlier recruitment of collagen fibers, as documented recently by Fonck and colleagues ${ }^{27}$ for elastase-treated carotid arteries through a microstructure-based biomechanical model. No regional differences in composition were found that might be associated with the absence of biomechanical variations in the circumferential direction, where histology was carried out. In view, however, of the presently reported changes in biomechanical properties along the longitudinal direction, histologic analysis in longitudinal sections might shed light on the local changes that take place in the presence of ATAA.

Our data indicated that aneurysms had no weakening effect on tissue strength, providing important information for better understanding the mechanism of ATAA rupture. Weak negative correlations were evidenced between maximum ATAA diameter and tensile strength, which is similar 
to our recent study. ${ }^{13}$ Today, maximum aneurysm diameter is the only universally acknowledged criterion considered for surgical intervention. ${ }^{4,5}$ Our results and similar ones reported by Vorp and coworkers ${ }^{9}$ indicated that this might not be the most representative parameter for the quality of aortic tissue. A possible explanation as to the rupture of ATAA, while being equally strong as for the nonaneurysmal aorta or even stronger, could then be ascribed to the increased wall stresses caused by tissue stiffening and vessel enlargement. Furthermore, the reduced tensile strength of the anterior region in the longitudinal direction associates closely with the clinical observation of preferential bulging occurring in that region, whereas the increased strength of the right lateral region suggests that this exhibits reinforced structure, despite being the most common site of aneurysm rupture. ${ }^{28,29}$

There are currently no detailed studies to report on the biomechanical behavior of the different aneurysmal layers, either in AAAs or ATAAs. Instead, all past studies have considered the wall to be homogenous across its thickness, an assumption that might lead to nonaccurate estimations of tissue strength and predictions of local stress distributions by using stress analysis models. In this study it was found that the different layers separated through testing and that the inner layers ruptured first, followed by the outer layers, which is indicative of layer-specific heterogeneity in strength and stiffness, but no mechanical testing on the individual layers was performed. We only examined histologic heterogeneity, quantifying histomorphometric and compositional parameters of the individual wall layers, and found the effect of ATAA to differ on each layer. Knowledge of the layer-specific properties of ATAA is, however, essential, because it is generally acknowledged that ATAA dissection is caused by a circumferential intimal tear. ${ }^{28-30}$

In conclusion, we present biomechanical evidence that ATAA development is not associated with mechanical weakening but with stiffening and reduction in vessel extensibility; histologic substantiation is provided. We also show that healthy and ATAA tissue exhibits similar regional heterogeneity and anisotropy. The present findings might help to better understand the pathogenesis of ATAA dissection, offering intuition for the development of computational models that will allow improved assessment of ATAA rupture potential.

\section{References}

1. Svensson LG, Crawford ES, eds. Cardiovascular and vascular disease of the aorta. Philadelphia: WB Saunders Company; 1997.

2. Coady MA, Rizzo JA, Goldstein LJ, Elefteriades JA. Natural history, pathogenesis and etiology of thoracic aortic aneurysms and dissections. Cardiol Clin. 1999; 17:615-35.

3. Juvonen T, Ergin MA, Galla JD, Lansman SL, McCullough JN, Levy D, et al. Prospective study of the natural history of thoracic aortic aneurysms. Ann Thorac Surg. 1997;63:1533-45.

4. Coady MA, Rizzo JA, Hammond GL, Mandapati D, Darr U, Kopf GS, et al. What is the appropriate size criterion for resection of thoracic aortic aneurysms? J Thorac Cardiovasc Surg. 1997;113:476-91.
5. Davies RR, Goldstein LJ, Coady MA, Tittle SL, Rizzo JA, Kopf GS, et al. Yearly rupture or dissection rates for thoracic aortic aneurysms: simple prediction based on size. Ann Thorac Surg. 2002;73:17-27.

6. Vorp DA, Vande Geest JP. Biomechanical determinants of abdominal aortic aneurysm rupture. Arterioscler Thromb Vasc Biol. 2005;25:1558-66.

7. Koullias G, Modak R, Tranquilli M, Korkolis DP, Barash P, Elefteriades JA. Mechanical deterioration underlies malignant behavior of aneurysmal human ascending aorta. J Thorac Cardiovasc Surg. 2005;130:677-83.

8. Okamoto RJ, Wagenseil JE, DeLong WR, Peterson SJ, Kouchoukos NT. Sundt TM III. Mechanical properties of dilated human ascending aorta. Ann Biomed Eng. 2002;30:624-35.

9. Vorp DA, Schiro BJ, Ehrlich MP, Juvonen TS, Ergin MA, Griffith BP. Effect of aneurysm on the tensile strength and biomechanical behaviour of the ascending thoracic aorta. Ann Thorac Surg. 2003;75:1210-4.

10. Groenink M, Langerak SE, Vanbavel E, van der Wall EE, Mulder BJM, van der Wal AC, et al. The influence of aging and aortic stiffness on permanent dilation and breaking stress of the thoracic descending aorta. Cardiovasc Res. 1999;43:471-80.

11. Mohan D, Melvin JW. Failure properties of passive human aortic tissue. I-uniaxial tension tests. J Biomech. 1982;15:887-902.

12. Tang PC, Coady MA, Lovoulos C, Dardik A, Aslan M, Elefteriades JA, et al. Hyperplastic cellular remodeling of the media in ascending thoracic aortic aneurysms. Circulation. 2005;112:1098-105.

13. Iliopoulos DC, Deveja RP, Kritharis EP, Perrea D, Sionis GD, Toutouzas K, et al Regional and directional variations in the mechanical properties of ascending thoracic aortic aneurysms. Med Eng Phys. 2008. Apr 21 [epub] In press.

14. Sokolis DP. Passive mechanical properties and structure of the aorta: segmental analysis. Acta Physiol (Oxf). 2007;190:277-89.

15. Mohan D, Melvin JW. Failure properties of passive human aortic tissue. II-Biaxial tension tests. J Biomech. 1983;16:31-44.

16. Okamoto RJ, Xu H, Kouchoukos NT, Moon MR. Sundt TM III. The influence of mechanical properties on wall stress and distensibility of the dilated ascending aorta. J Thorac Cardiovasc Surg. 2003;126:842-50.

17. Mitchell GF, Parise H, Benjamin EJ, Larson MG, Keyes MJ, Vita JA, et al. Changes in the arterial stiffness and wave reflection with advancing age in healthy men and women: the Framingham Heart Study. Hypertension. 2004;43:1239-45.

18. Raghavan ML, Webster MW, Vorp DA. Ex vivo biomechanical behavior of abdominal aortic aneurysm: assessment using a new mathematical model. Ann Biomed Eng. 1996;24:573-82.

19. Vallabhanemi SR, Gilling-Smith GL, How TV, Carter SD, Brennan JA, Harris PL. Heterogeneity of tensile strength and matrix metalloproteinase activity in the wall of abdominal aortic aneurysms. J Endovasc Ther. 2004;11: 494-502.

20. Vande Geest JP, Sacks MS, Vorp DA. The effects of aneurysm on the biaxial mechanical behavior of human abdominal aorta. J Biomech. 2006;39:1324-34.

21. Marra SP, Kennedy FE, Kinkaid JN, Fillinger MF. Elastic and rupture properties of porcine aortic tissue measured using inflation testing. Cardiovasc Eng. 2006;6: 125-33.

22. Nicosia MA, Kasalko JS, Cochran RP, Einstein DR, Kunzelman KS. Biaxial mechanical properties of porcine ascending aortic wall tissue. J Heart Valve Dis. 2002;11:680-6.

23. Thubrikar MJ, Labrose M, Robicsek F, Al-Soudi J, Fowler B. Mechanical properties of abdominal aortic aneurysm wall. J Med Eng Technol. 2001;25:133-42.

24. He CM, Roach MR. The composition and mechanical properties of abdominal aortic aneurysms. J Vasc Surg. 1994;20:6-13.

25. Dobrin PB. Pathophysiology and pathogenesis of aortic aneurysms. Current concepts. Surg Clin North Am. 1989;69:687-703.

26. Sokolis DP, Kefaloyannis EM, Kouloukoussa M, Marinos E, Boudoulas H, Karayannacos PE. A structural basis for the aortic stress-strain relation in uniaxial tension. J Biomech. 2006;39:1651-62.

27. Fonck E, Prod'hom G, Roy S, Augsburger L, Rufenacht DA, Stergiopulos N. Effect of elastin degradation on carotid wall mechanics as assessed by a constituent-based biomechanical model. Am J Physiol Heart Circ Physiol. 2007;292:H2754-63.

28. Black JH III, Cambria RP. Aortic dissection: perspectives for the vascular/endovascular surgeon. In: Cronenwett JL, Gloviczki P, Johnston KW, Krupski WC, Ouriel K, eds. Rutherford: vascular surgery. 6th ed. Philadelphia: WB Saunders $\mathrm{Co} ; 2005$.

29. Beller CJ, Labrosse MR, Thubrikar MJ, Robicsek F. Role of aortic root motion in the pathogenesis of aortic dissection. Circulation. 2004;109:763-9.

30. Thubrikar MJ, Agali P, Robicsek F. Wall stresses as a possible mechanism for development of transverse intimal tears in aortic dissections. J Med Eng Technol. 1999;23:127-34. 